\title{
Effect of a carbohydrate-electrolyte drink on endurance capacity during prolonged intermittent high intensity running
}

George P Nassis, Clyde Williams, Penelope Chisnall

\begin{abstract}
Objective-To examine the effect of a carbohydrate-electrolyte solution on endurance capacity during prolonged intermittent running.

Methods-Nine subjects (eight men and one woman) ran to exhaustion on a motorised treadmill on two occasions separated by at least 10 days. After an overnight fast, they performed repeated 15 second bouts of fast running (at $80 \%$ Vo,MAX for the first 60 minutes, at $85 \%$ Vo $0_{2}$ AX from 60 to 100 minutes of exercise, and finally at $\mathbf{9 0 \%} \mathrm{VO}_{2} \mathrm{MAx}$ from 100 minutes of exercise until exhaustion), separated by 10 seconds of slow running (at $\left.45 \% \mathrm{Vo}_{2} \mathrm{MAx}\right)$. On each occasion they drank either a water placebo (P) or a $6.9 \%$ carbohydrate-electrolyte (CHO) solution immediately before the run $(3 \mathrm{ml} / \mathrm{kg}$ body mass) and every 20 minutes thereafter (2 $\mathrm{ml} / \mathrm{kg}$ body mass).

Results-Performance times were not different between the two trials (112.5 (23.3) and $110.2(21.4) \mathrm{min}$ for the $P$ and CHO trials respectively; mean (SD)). Blood glucose concentration was higher in the CHO trial only at 40 minutes of exercise (4.5 (0.6) $v 3.9(0.3) \mathrm{mmol} / 1$ for the CHO and $P$ trials respectively; $p<0.05)$, but there was no difference in the total carbohydrate oxidation rates between trials.

Conclusion-These results suggest that drinking a $6.9 \%$ carbohydrate-electrolyte solution during repeated bouts of submaximal intermittent high intensity running does not delay the onset of fatigue. (Br F Sports Med 1998;32:248-252)
\end{abstract}

Keywords: intermittent exercise; running; fluid intake; endurance capacity

Department of Physical Education, Sports Science, and Recreation

Management,

Loughborough

University,

Loughborough, Leics

LE11 3TU, United

Kingdom

G P Nassis

C Williams

P Chisnall

Correspondence to: Professor C Williams.

Accepted for publication 7 April 1998 during continuous submaximal exercise also delays the onset of fatigue..$^{5-8}$ The reasons suggested for the improvement in endurance capacity are: (a) prevention of hypoglycaemia ${ }^{2}$ and $(b)$ maintenance of a high rate of carbohydrate oxidation throughout exercise. ${ }^{2} 7$ There is also some evidence to suggest that the improvement in endurance capacity is a consequence of glycogen sparing. ${ }^{9} 10$

Improvements in performance can also be achieved during intermittent exercise when carbohydrate solutions are consumed throughout exercise. ${ }^{11-14}$ In these studies on intermittent exercise, cycling was the mode of exercise. Only a limited number of studies have examined the effect of drinking carbohydrate solutions on performance during intermittent running. ${ }^{15} 16$ Therefore the aim of this study was to investigate the influence of drinking a carbohydrate-electrolyte solution on endurance capacity during prolonged intermittent high intensity running.

\section{Methods}

SUBJECTS

Nine subjects (eight men and one woman) gave their informed consent and volunteered to participate in this study which had the approval of the University ethical committee. Five of them were club level runners whereas the remainder were ex-club level runners. All subjects were experienced endurance trained athletes. The mean (SD) values for age, height, body mass, and maximal oxygen uptake $\left(\mathrm{VO}_{2} \mathrm{MAx}\right)$ of the group were $25(4.3)$ years, $176(7.0) \mathrm{cm}, 72.8$ $(10.5) \mathrm{kg}$, and $4.69(0.59)$ litres/min (65.1 (8.8) $\mathrm{ml} / \mathrm{kg} / \mathrm{min}$ ).

\section{PRELIMINARY MEASUREMENTS}

A 16 minute continuous submaximal running test was used to determine the relation between running speed and oxygen cost for each subject on a motorised treadmill (Woodway ELG 2; Woodway Geres $\mathrm{GmbH}$, Weil am Rhein, Germany). $\mathrm{VO}_{2} \mathrm{MAX}$ was determined during uphill treadmill running by employing a continuous incremental test to exhaustion. ${ }^{17}$ Using the results from these two tests, the running speeds equivalent to $45,60,70,80,85$, and $90 \% \mathrm{VO}_{2} \mathrm{MAX}$ were predicted. To verify the accuracy of these predictions the subjects ran on the level treadmill for four minutes at each of four speeds corresponding to $60,70,80$, and $90 \% \mathrm{VO}_{2} \mathrm{MAX}$. Finally, they completed a 30 minute intermittent treadmill run, similar to that performed during the experimental trials, in order to familiarise themselves with the running speeds, the rate of change in speed, and the experimental procedures.
EXPERIMENTAL DESIGN

The subjects performed two experimental trials separated by at least 10 days. To eliminate any order or training effect, the order of trials 
was randomly assigned. On each occasion, subjects consumed either a water placebo artificially sweetened with aspartame $(\mathrm{P})$ or a $6.9 \%$ commercially available carbohydrateelectrolyte solution (CHO) (Lucozade-Sport; SmithKline Beecham Coleford, Glos, UK) immediately before the start of the run $(3 \mathrm{ml} / \mathrm{kg}$ body mass) and every 20 minutes during exercise $(2 \mathrm{ml} \mathrm{kg} /$ body mass $)$. The solutions ingested were of the same colour, texture, and taste, and were administered in a double blind design. They were kept in the refrigerator, removed about 10 minutes before consumption, and warmed up to a constant temperature of $8-9^{\circ} \mathrm{C}$. Drinks were taken from plastic $30 \mathrm{ml}$ syringes to prevent spillage.

The subjects were asked to refrain from heavy exercise for two days before each trial and record their normal diet for three days preceding the first trial. They were required to replicate exactly the same diet for the same period of time before the second trial.

On the day of the experimental trial each subject arrived in the laboratory early in the morning after an overnight fast of 10 to 12 hours. The nude body mass was recorded for each subject after they had emptied their bladders. The subject was then asked to assume an upright position. After a 20 minute period in the standing position, samples of arterialised capillary blood were taken before a 10 minute standardised warm up. The warm up consisted of five minutes continuous treadmill running at $60 \% \mathrm{Vo}_{2} \mathrm{MAX}$ followed by five minutes intermittent running (repeated 15 second bouts at $70 \%$ $\mathrm{VO}_{2} \mathrm{MAX}$ interrupted by 10 second bouts at $45 \%$ $\left.\mathrm{VO}_{2} \mathrm{MAX}\right)$. An expired air sample was collected during the final 50 seconds of the warm up using the Douglas bag method. The treadmill was then stopped for five minutes during which period blood samples were obtained and the subjects consumed $3 \mathrm{ml} / \mathrm{kg}$ body mass of the assigned fluid while standing on the treadmill.

Immediately after this five minute period, the intermittent running protocol was initiated. During the experimental trials, the subjects completed repeated 15 second bouts of fast running followed by 10 seconds of running at slower speeds. This protocol was first described by Bangsbo and colleagues. ${ }^{18}$ The speed for the 10 second bouts corresponded to $45 \% \mathrm{VO}_{2} \mathrm{MAX}$ for the entire run whereas the speed for the 15 second bouts varied, being equivalent to $80 \%$ $\mathrm{VO}_{2} \mathrm{MAX}$ for the first 60 minutes, $85 \% \mathrm{Vo}_{2} \mathrm{MAX}$ from 60 to 100 minutes, and finally $90 \%$ $\mathrm{VO}_{2} \mathrm{MAX}$ from 100 minutes until volitional fatigue. The changes in running speed were initiated and controlled by a micro-computer (BBC model B) interfaced with the treadmill, and programmed to change the belt speed at appropriate times, which took about two seconds. Fatigue was defined as the inability of subjects to maintain the prescribed running speed for two consecutive bouts of fast running. Subjects were verbally encouraged in both trials and were not informed of their performance times until the completion of the study.
PHYSIOLOGICAL MEASUREMENTS

Expired air samples were collected in Douglas bags for 50 seconds during the last minute of the warm up, every 20 minutes during the run, and during the final minute of the run. Sampling was always initiated at the beginning of the low speed running bouts. Samples of expired air were analysed for oxygen (Sybron/ Taylor 570A; Servomex, Crowborough, Sussex, UK) and carbon dioxide content (Lira 3000; MSA, East Shawhead, Coatbridge, Scotland, UK), total volume, and temperature (Harvard Apparatus, Edenbridge, Kent,UK). ${ }^{19}$ This allowed the calculation of $\mathrm{VO}_{2}$, carbon dioxide production, and respiratory exchange ratio (RER). To ascertain the subjects' perception of fatigue, the Borg Scale ${ }^{20}$ was shown to them during the collection of expired air samples. Heart rates were monitored throughout each run on an oscilloscope (Rigel Ltd, Morden, Surrey, UK) from four chest electrodes, and the values for the fast and the slow running bouts were recorded for one minute every five minutes. From these, the average values for each 20 minute period were calculated.

BLOOD SAMPLING AND ANALYSIS

Duplicate $20 \mu 1$ samples of arterialised capillary blood were taken from the thumb of a prewarmed hand before exercise and after the warm up ( $0 \mathrm{~min})$, every 20 minutes throughout the run, and immediately after the completion of exercise, for blood glucose and lactate analyses. Blood samples were immediately deproteinised in a solution containing $200 \mu \mathrm{l} 2.5 \%$ perchloric acid. These samples were then frozen at $-20^{\circ} \mathrm{C}$ and were later analysed in duplicate for blood glucose and lactate concentrations. ${ }^{21}$ Samples were obtained before and after exercise while the subjects were standing on the treadmill. All other samples were taken while subjects were running. Blood sampling during exercise was always initiated after the completion of expired air collection and without interrupting the running speed of the subjects.

\section{SWEAT RATE AND CARBOHYDRATE OXIDATION} RATE

Sweat rate (litres/hour) was calculated as change in nude body mass corrected for respiratory water loss, metabolic fuel utilised, and fluid intake. ${ }^{22}$ Nude body mass was recorded before the warm up and immediately after the run after subjects had removed the sweat from the skin and had urinated. Respiratory water loss was calculated as $0.026 \mathrm{~g} / \mathrm{kJ} .{ }^{23}$ Metabolic fuel utilised (g) was calculated from the total energy expenditure $(\mathrm{kJ})$ during the trials and converted into grams of fuel oxidised minus the metabolic water (g) released from the fuel oxidation. ${ }^{24}$ For these calculations it was assumed that $0.55 \mathrm{~g}$ water is released/g carbohydrate oxidised and $1.07 \mathrm{~g}$ water is released/g fat oxidised. ${ }^{24}$ Dry bulb temperatures within the laboratory were $21.6(1.8)$ and 21.6 (1.6) ${ }^{\circ} \mathrm{C}$ during the $\mathrm{CHO}$ and $\mathrm{P}$ trials respectively. Relative humidity was $63(8) \%$ during the $\mathrm{CHO}$ and $66(10.3) \%$ during the $\mathrm{P}$ trial 


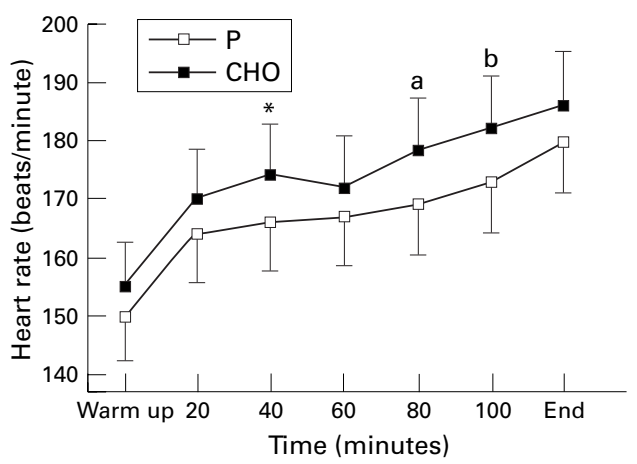

Figure 1 Heart rate responses (beats/minute) for the placebo $(P)$ and carbohydrate-electrolyte (CHO) trials during intermittent running to exhaustion (mean (SD); $n$ $=9$ except for the point marked a where $n=8$ and that marked $b$ where $n=6$ ). ${ }^{\star}$ Significantly different from $P$ trial; $p<0.05$.

(not significantly different). Finally, the total carbohydrate oxidation rate was estimated from RER and $\mathrm{VO}_{2}$ values.

\section{STATISTICAL ANALYSIS}

Differences in performance times, distances covered, environmental temperatures, changes in body mass, and volume of fluid ingested over the two experimental trials were examined using Student's $t$ test for correlated samples. Statistical comparisons of the physiological and metabolic parameters between the $\mathrm{CHO}$ and $\mathrm{P}$ trials were undertaken for the sampling points where complete data were available - that is, rest before exercise, 0, 20, 40, 60 minutes and at exhaustion-using a two way analysis of variance for repeated measures on two factors (experimental condition and sampling time). Where significant differences were found, the Tukey test was employed to identify the differences. Statistical significance was accepted at the 0.05 level. Values are presented as mean (SD).

\section{Results}

RUNNING PERFORMANCE

The performance times for the $\mathrm{P}$ and the $\mathrm{CHO}$ trials were 112.5 (23.3) and 110.2 (21.4) minutes respectively (not significantly different). One subject reached fatigue before 80 minutes in both trials. Six subjects completed $100 \mathrm{~min}-$ utes of exercise in both trials whereas only three in the CHO and four in the P trial completed 120 minutes of running. The distances covered during the experimental trials were 25.0 (6.4) and 22.9 (9.3) $\mathrm{km}$ for the $\mathrm{P}$ and the $\mathrm{CHO}$ trial respectively (difference not significant). There was no order effect for running times between the first and second trial. Finally, the speeds corresponding to $45,80,85$, and $90 \% \mathrm{VO}_{2} \mathrm{MAX}$ for the subjects averaged 2.62 (0.43), 4.30 (0.53), 4.55 (0.55), and 4.79 $(0.56) \mathrm{m} / \mathrm{s}$ respectively.

HEART RATE AND PERCEIVED RATE OF EXERTION There was a trend for the heart rates to be higher in the $\mathrm{CHO}$ trial, although the only significant difference was found at 40 minutes of exercise $(\mathrm{p}<0.05$; fig 1$)$. Perceived rate of exer-

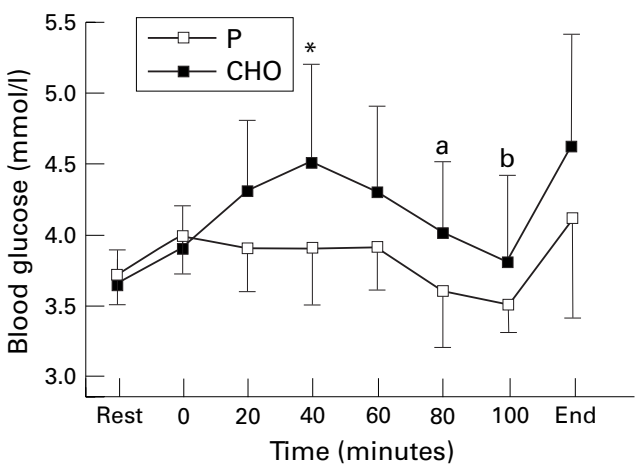

Figure 2 Blood glucose concentrations (mmol/l) for the placebo $(P)$ and carbohydrate-electrolyte (CHO) trials during intermittent running to exhaustion (mean (SD);n $=9$ except for the point marked $a$ where $n=8$ and that marked $b$ where $n=6$ ). * Significantly different from $P$ trial; $p<0.05$.

tion was not different between the experimental runs, reaching a value of 19 at the end of exercise regardless of condition.

OXYGEN UPTAKE

The average oxygen uptake during the $\mathrm{P}$ and CHO trials was $3.52(0.54)$ and $3.48(0.54)$ litres/min respectively (not significantly different). These values represent average relative exercise intensities of $75 \quad(3.0) \% \quad \mathrm{VO}_{2} \mathrm{MAX}$ during the $\mathrm{P}$ trial and $74.0(4.2) \% \mathrm{VO}_{2} \mathrm{MAX}$ during the $\mathrm{CHO}$ trial. Oxygen uptake gradually increased throughout exercise in both trials, reaching values of $3.39(0.52)$ and 3.29 (0.41) litres/min at 60 minutes of exercise for the $\mathrm{P}$ and $\mathrm{CHO}$ trials respectively - that is, $72.3(3.6) \%$ and 70 (5.7)\% $\mathrm{VO}_{2} \mathrm{MAX}$ respectively. At 100 minutes of exercise, $\mathrm{VO}_{2}$ was 3.62 (0.84) litres/min (76.8 (3.6)\% $\left.\mathrm{Vo}_{2} \mathrm{MAX}\right)$ for the $\mathrm{P}$ and 3.57 (0.84) litres/min (75.9 (8.4)\% $\left.\mathrm{VO}_{2} \mathrm{MAX}\right)$ for the $\mathrm{CHO}$ trial whereas during the final minute of the run these values were 3.72 (0.57) litres/min (78.8 (2.7)\% $\left.\mathrm{VO}_{2} \mathrm{MAX}\right)$ and 3.69 (0.54) litres/min (78.6 (7.5)\% $\left.\mathrm{VO}_{2} \mathrm{MAX}\right)$ for the $\mathrm{P}$ and $\mathrm{CHO}$ trials respectively.

RATE OF CARBOHYDRATE OXIDATION

The average carbohydrate oxidation rates were similar for the two trials $(2.4(0.2)$ and 2.2 (0.1) $\mathrm{g} / \mathrm{min}$ for the $\mathrm{CHO}$ and $\mathrm{P}$ trial respectively).

SWEAT RATE AND BODY MASS

The total volume of fluid ingested was 877 (98) and 906 (143) $\mathrm{ml}$ in the $\mathrm{CHO}$ and P trials respectively. As a result of sweating, body mass, corrected for fluid intake and urine volume, decreased by $2.8(0.8) \mathrm{kg}$ in both trials. Thus the average sweat rate was similar in the two conditions (1.5 (0.3) litres/hour).

BLOOD GLUCOSE AND LACTATE CONCENTRATIONS Resting blood glucose concentration was similar in the two trials (fig 2); a difference was found only at 40 minutes of exercise $(\mathrm{p}<0.05)$. In the $\mathrm{CHO}$ trial, blood glucose concentrations were higher than the resting values at $20 \mathrm{~min}$ utes $(p<0.05), 40$ minutes $(p<0.01), 60$ minutes $(p<0.01)$ of exercise, and at the point of exhaustion $(\mathrm{p}<0.01)$. In contrast, during the 


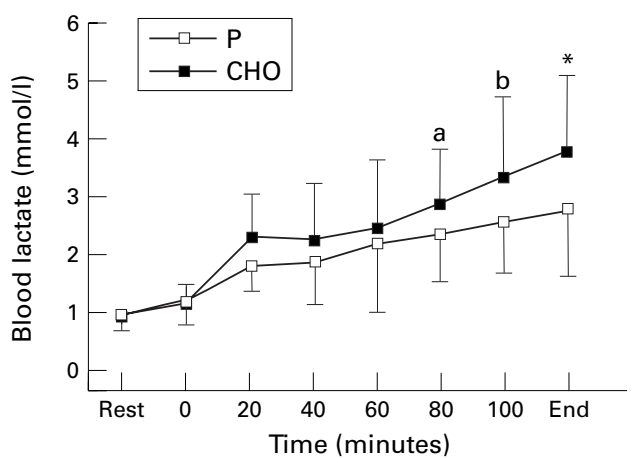

Figure 3 Blood lactate concentrations ( $\mathrm{mmol} / \mathrm{l}$ ) for the placebo $(P)$ and carbohydrate-electrolyte $(\mathrm{CHO})$ trials during intermittent running to exhaustion (mean (SD);n $=9$ except for the point marked $a$ where $n=8$ and that marked $b$ where $n=6$ ). ${ }^{\star}$ Significantly different from $P$ trial; $p<0.05$.

$\mathrm{P}$ trial blood glucose concentrations did not differ from the resting values except at the point of exhaustion $(\mathrm{p}<0.01)$.

Blood lactate concentrations measured at rest and after the warm up were similar in the two trials (fig 3). In the CHO trial, blood lactate concentration gradually increased and the values at all the sampling points were significantly above the resting value $(p<0.01)$. A significant difference between trials was found only at exhaustion $(p<0.05)$.

\section{Discussion}

The main finding of this study is that the ingestion of a $6.9 \%$ carbohydrate-electrolyte solution did not improve endurance capacity during prolonged intermittent high intensity running. Performance times for the $\mathrm{CHO}$ and $\mathrm{P}$ trials were similar even though the runners ingested the equivalent of $30 \mathrm{~g} / \mathrm{h}$ carbohydrate throughout the $\mathrm{CHO}$ trial.

The failure of the ingested carbohydrateelectrolyte solution to delay the onset of fatigue is in marked contrast with the results of studies on constant load cycling ${ }^{25}$ and constant pace treadmill running, ${ }^{81026}$ which report improvements in endurance capacity. Performance during prolonged intermittent high intensity cycling $^{911} 1327$ and running ${ }^{1516}$ also benefits from drinking carbohydrate-electrolyte solutions throughout exercise. In all these studies, the brief periods of high intensity exercise were separated by several minutes of low intensity exercise or rest. In this respect, the physical demands on the subjects in the present study were far greater than experienced by subjects in most of the previously reported studies on intermittent high intensity submaximal exercise.

The intermittent high and low speed treadmill running protocol used in the present study was the same as that used by Bangsbo et $a l^{18}$ to examine the influence of carbohydrate nutrition on performance during an activity pattern which is common in soccer. In their study the professional soccer players undertook dietary carbohydrate loading for two days before performing the exercise tests. Immediately before they undertook the treadmill multiple sprint test, the soccer players performed 46 minutes of field exercises, which involved some callisthenics and some intermittent running. Thereafter, they performed the treadmill multiple sprint test to the point of fatigue. After carbohydrate loading, the soccer players ran $17.1 \mathrm{~km}$ which was $0.9 \mathrm{~km}$ further than the distance they covered when they were on their normal mixed diet. ${ }^{18}$

The distances covered by the runners in the present study were 25.0 and $22.9 \mathrm{~km}$ for the $\mathrm{P}$ and $\mathrm{CHO}$ trials respectively. The reasons for the difference in the distances covered in the two studies are $(a)$ the subjects in the present study had higher $\mathrm{VO}_{2} \mathrm{MAX}$ values (65 $v 60$ $\mathrm{ml} / \mathrm{kg} / \mathrm{min}$ ) and so their prescribed running speeds were faster than those of the soccer players in the study of Bangsbo et al, and $(b)$ the soccer players completed 46 minutes of exercise before performing the treadmill multiple sprint test, which probably contributed to the earlier onset of fatigue. ${ }^{18}$

Although pre-exercise muscle glycogen concentrations were not reported in the study by Bangsbo et $a l,{ }^{18}$ it is reasonable to assume that the carbohydrate stores of soccer players were well stocked before they undertook the exercise tests. The subjects in the present study did not undertake dietary carbohydrate loading before performing the treadmill multiple sprint test, but carbohydrate was provided throughout exercise. Therefore one explanation for the lack of improvement in performance may be the inadequate delivery of a relatively modest amount of carbohydrate $(30 \mathrm{~g} / \mathrm{h})$ to working muscles as a consequence of a reduction in the rate of gastric emptying.

Gastric emptying of carbohydrate-electrolyte solutions does not appear to be severely impaired by constant pace exercise at intensities lower than about $70 \% \mathrm{VO}_{2} \mathrm{MAX} .{ }^{28}$ However, the overall exercise intensity was greater than $70 \% \mathrm{VO}_{2} \mathrm{MAX}$ in the present study. Furthermore, the repeated changes from low to high intensity exercise along with the increase in speed as the test progressed will also have contributed to a decrease in the rate of gastric emptying. Three observations suggest that the runners in this study did not fully benefit from drinking the carbohydrate-electrolyte solution. The first is that blood glucose concentrations were, with one exception, not significantly different between trials; the second is that, other than at the point of fatigue, the blood lactate concentrations were also similar in the two trials. Thirdly, there were no differences in the RER values between the two trials. Of course, RER values reliably reflect muscle metabolism only during prolonged constant pace submaximal exercise. However, it is reasonable to assume that even though the exercise was intermittent in nature, the interval between high and low speed running was short and the overall duration was such that a near steady state in oxygen uptake and carbon dioxide production will have been reached after an hour or more of this exercise. ${ }^{29} 30$ Accepting that accurate estimates of substrate oxidation rates cannot be derived from the RER values under these conditions, nevertheless, it is reasonable to conclude from the similarity of the RER val- 
ues that there was no difference between the carbohydrate oxidation rates in the two trials. Thus there was no clear evidence that the runners oxidised a greater amount of carbohydrate during the $\mathrm{CHO}$ trial than they did during the $P$ trial.

The runners in the present study performed about 265 repeated periods of high - that is, equal to at least $80 \% \mathrm{VO}_{2} \mathrm{MAX}$ - and low intensity running, separated by only 10 seconds. This prolonged intermittent high intensity exercise probably made large demands on the runners' muscle glycogen stores. In a study on muscle metabolism during 90 minutes of intermittent high intensity shuttle running, we found that there were greater reductions in the glycogen concentrations in the type 2 muscle fibres (fast contracting fast fatiguing muscle fibres) than in the type 1 muscle fibres (slow contracting slow fatiguing) (C W Nicholas, $\mathrm{K} \mathrm{O}$ Tsinatz, L H Boobis, et al, unpublished work). This may explain why, towards the end of the tests, the subjects had difficulty in accelerating with the change in treadmill speed. Furthermore, progressive failure of the active muscle fibres to resynthesise sufficient phosphocreatine during the brief recovery periods of low intensity running will also have contributed to the inability of the runners to cope with the changes in treadmill speed. Repeated high intensity exercise with 30 seconds or less of resting recovery leads to a gradual decrease in human power output, which is accompanied by a decrease in phosphocreatine concentration. $^{31}{ }^{32}$ Thus it is reasonable to conclude that the fatigue that occurred after nearly two hours of intermittent high intensity running was a consequence of a reduction in muscle glycogen concentration and an inadequate rate of phosphocreatine resynthesis. ${ }^{33}$ G N was a scholar of the State Scholarship Foundation of
Greece during the course of this project. The authors would like to thank Dr Jens Bangsbo and Dr Rabindarjeet Singh for their to thank Dr Jens Bangsbo and Dr Rabindarjeet Singh for their
invaluable assistance in designing the experimental protocol, Dr invaluable assistance in designing the experimental protocol, Dr $\mathrm{H}$ K A Lakomy who developed the software for the intermittent
runs, and finally Dr K O Tsintzas for his expert assistance on runs, and finally $\mathrm{D}$
experimental days.

1 Bergstrom J, Hermansen L, Hultman E, et al. Diet, muscle glycogen and physical performance. Acta Physiol Scand $1967 ; 71: 140-50$

2 Coyle EF, Coggan AR, Hemmert MK, et al. Muscle glycogen utilization during prolonged strenuous exercise when fed carbohydrate. F Appl Physiol 1986;61:165-72.

3 Armstrong L, Costill D, Fink W. Influence of diuretic induced dehydration on competitive running performance. Med Sci Sports Exerc 1985;17:456-61.

4 Montain S, Coyle E. Influence of graded dehydration on hyperthermia and cardiovascular drift during exercise. $f$ Appl Physiol 1992;73:1340-50.

5 Ivy J, Costill D, Fink W, et al. Influence of caffeine and carbohydrate feedings on endurance performance. Med $\mathrm{Sc}$ Sports Exerc 1979;11:6-11.

6 Coyle E, Hagberg J, Hurley B, et al. Carbohydrate feeding during prolonged strenuous exercise can delay fatigue. $\mathcal{F}$ Appl Physiol 1983;55:230-5.
7 Coggan A, Coyle E. Metabolism and performance following carbohydrate ingestion late in exercise. Med Sci Sports Exerc 1989;21:59-65.

8 Tsintzas KO, Williams C, Wilson W, et al. Influence of carbohydrate supplementation early in exercise on endurance running capacity. Med Sci Sports Exerc 1996;28:1373-9.

9 Yaspelkis B, Patterson J, Anderla P, et al. Carbohydrate supplementation spares muscle glycogen during variableintensity exercise. F Appl Phsiol 1993;75:1477-85.

10 Tsintzas KO, Williams C, Boobis L, et al. Carbohydrate ingestion and single muscle fiber glycogen metabolism during prolonged running in men. $\mathcal{f}$ Appl Physiol 1996;81: $801-9$.

11 Fielding R, Costill D, Fink W, et al. Effect of carbohydrate feeding frequencies and dosage on muscle glycogen use during exercise. Med Sci Sports Exerc 1985;17:472-6.

12 Murray R, Eddy D, Murray W, et al. The effect of fluid and carbohydrate feedings during intermittent cycling exercise. Med Sci Sports Exerc 1987;19:597-603.

13 Hargreaves M, Costill D, Coggan A, et al. Effect of carbohydrate feedings on muscle glycogen utilization and exercise performance. Med Sci Sports Exerc 1984;16:219-22.

14 Murray R, Paul G, Seifert J, et al. Responses to varying rates of carbohydrate ingestion during exercise. Med Sci Sports Exerc 1991;23:713-18.

15 Kirkendall D, Foster C, Dean J, et al. Effect of glucose polymer supplementation on performance of soccer players. In: Reilly T, Lees A, David K, Murphy W, eds. Science and football. London: E \& FN Spon, 1988:33-41.

16 Nicholas C, Williams C, Lakomy H, et al. Influence of ingesting a carbohydrate-electrolyte solution on endurance capacity during intermittent, high-intensity shuttle running. F Sports Sci 1995;13:283-90.

17 Taylor H, Buskirk E, Henschel A. Maximal oxygen intake as an objective measure of cardio-respiratory performance. $\mathcal{F}$ Appl Physiol 1955;8:73-80.

18 Bangsbo J, Norregaard L, Thorsoe F. The effect of carbohydrate diet on intermittent exercise performance. Int $\mathcal{F}$ Sports Med 1992;13:152-7.

19 Williams C, Nute M. Some physiological demands of a halfmarathon race on recreational runners. Br 7 Sports Med 1983;17:152-61.

20 Borg G. Perceived exertion: a note on 'history' and methods. Med Sci Sports Exerc 1973;5:90-3.

21 Maughan RJ. A simple, rapid method for the determination of glucose, lactate, pyruvate, alanine, 3-hydroxybutyrate and acetoacetate on a single $20 \mu \mathrm{l}$ blood sample. Clin Chim Acta 1982;122:231-40.

22 Noakes T, Adams B, Myburgh K, et al. The danger of inadequate water intake during prolonged exercise. A novel concept revisited. Eur f Appl Physiol 1988;57:210-20.

23 Pugh L, Corbett J, Johnson R. Rectal temperatures, weight losses and sweat rates in marathon running. $\mathcal{f}$ Appl Physiol 1967;23:347-52.

24 Noakes T, Myburgh K, Du Plessis J, et al. Metabolic rate, not percent dehydration, predicts rectal temperature in marathon runners. Med Sci Sports Exerc 1991;23:443-9.

25 Maughan R, Fenn C, Leiper J. Effects of fluid, electrolyte and substrate ingestion on endurance capacity. Eur $\mathcal{F} A p p l$ Physiol 1989;58:481-6.

26 Wilber R, Moffatt R. Influence of carbohydrate ingestion on blood glucose and performance in runners. Int $\mathcal{F}$ Sport Nutr 1992;2:317-27.

27 Murray R, Eddy DE, Murray T, et al. The effect of fluid and carbohydrate feedings during intermittent cycling exercise. Med Sci Sports Exerc 1987;19:597-604.

28 Brouns F, Saris W, Rehrer N. Abdominal complaints and gastrointestinal function during long-lasting exercise. Int $\mathcal{F}$ Sports Med 1987;8:175-89.

29 Essen B, Hagenfeldt L, Kaijser L. Utilization of blood-borne and intramuscular substrates during continuous and intermittent exercise in man 7 Physiol (Lond) 1977;265:489506.

30 Essen B. Studies on the regulation of metabolism in human skeletal muscle using intermittent exercise as an experimental model. Acta Physiol Scand 1978;454(suppl): 1-32.

31 Gaitanos G, Williams C, Boobis L, et al. Human muscle metabolism during intermittent maximal exercise. $\mathcal{F} A p p l$ Physiol 1993;75:712-19.

32 Balsom P, Seger J, Sjodin B, et al. Physiological responses to maximal intensity intermittent exercise. Eur f Appl Physiol 1992;65:144-9.

33 Bogdanis G, Nevill M, Boobis L, et al. Recovery of power output and muscle metabolites following $30 \mathrm{~s}$ of maximal sprint cycling in man. $\mathcal{F}$ Physiol (Lond) 1995;482:467-80 